\title{
Size-selective detection of nanoparticles in solution and air by imprinting
}

\author{
Linoy Dery, ${ }^{1, \mp}$ Nitzan Shauloff, ${ }^{2, ¥}$ Yury Turkulets, ${ }^{3}$ Ilan Shalish, ${ }^{3}$ Raz Jelinek, ${ }^{2, *}$ and Daniel
}

Mandler, ${ }^{1, *}$

1 Institute of Chemistry, the Hebrew University of Jerusalem, Jerusalem 9190401, Israel.

2 Ilse Katz Institute for Nanotechnology Ben Gurion University of the Negev Beer Sheva 8410501, Israel.

3 School of Electrical Engineering, Ben-Gurion University, Beer Sheva, 8410501 Israel.

$¥$ These authors contributed equally.

*Email: Daniel.mandler@mail.huji.ac.il and razj@bgu.ac.il
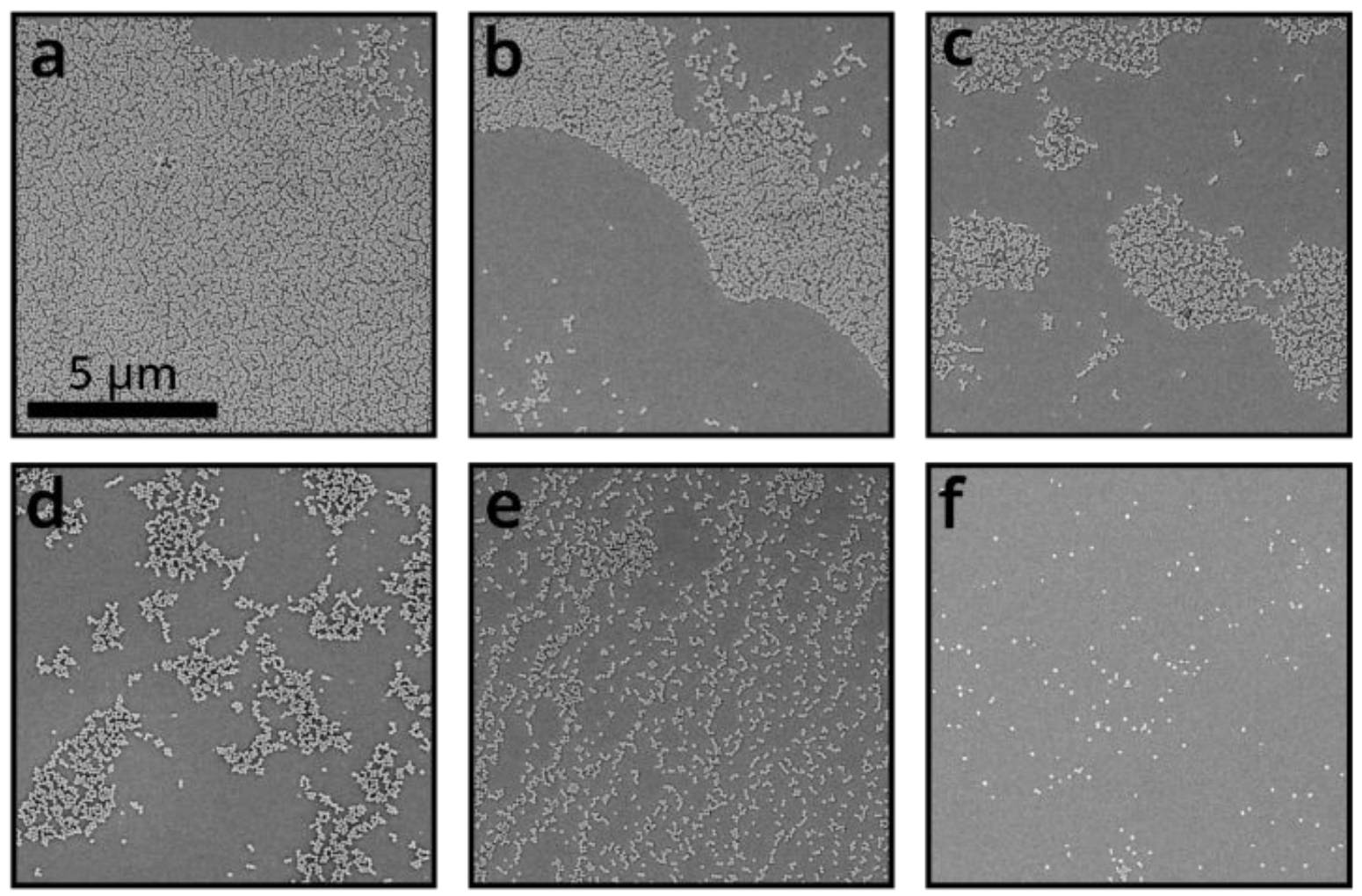

Figure S1. SEM images of spin-coated latex NPs dispersed on glass surfaces with different NP concentrations or spin-coating speeds. (a) $1 \mathrm{wt} \%$ of latex NPs at $2000 \mathrm{rpm}$ for $1 \mathrm{~min}$, (b) $0.75 \mathrm{wt} \%$ of latex NPs at $2000 \mathrm{rpm}$ for 1 min, (c) $0.75 \mathrm{wt} \%$ of latex NPs at $3000 \mathrm{rpm}$ for $1 \mathrm{~min}$, (d) $0.5 \mathrm{wt} \%$ of latex NPs at $2000 \mathrm{rpm}$, (e) $0.5 \mathrm{wt} \% \mathrm{of}$ latex 
NPs at $3000 \mathrm{rpm}$ for $1 \mathrm{~min}$, (f) $0.25 \mathrm{wt} \%$ of latex NPs at $3000 \mathrm{rpm}$ for $1 \mathrm{~min}$. All samples were spattered for 60 sec with a thin AuPd layer.
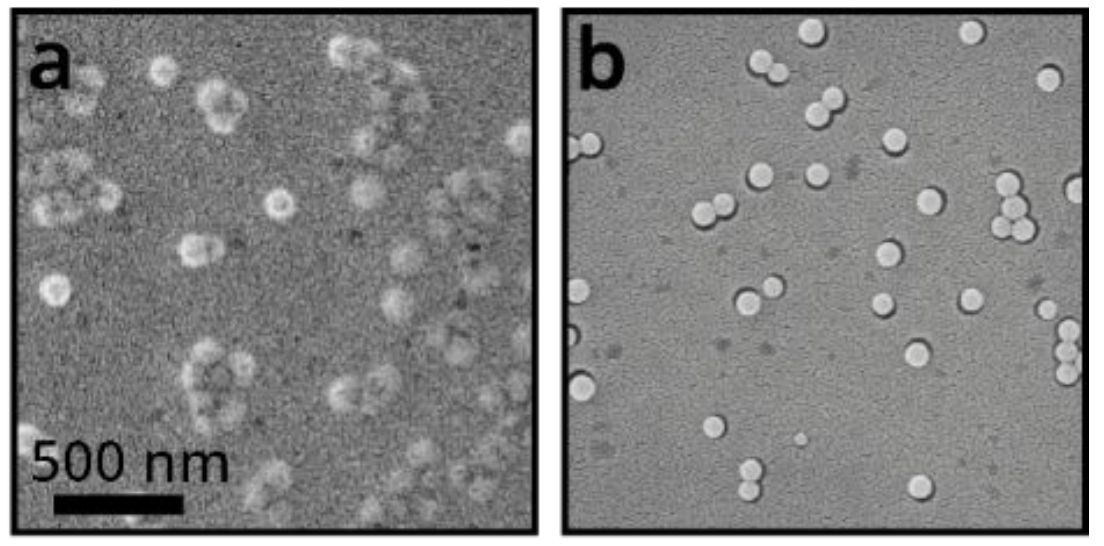

Figure S2. SEM images of latex NPs/sol-gel matrix spin-coated at: (a) 1500 and (b) $3000 \mathrm{rpm}$ for $1 \mathrm{~min}$. All samples were sputtered for $60 \mathrm{sec}$ with a thin AuPd layer.
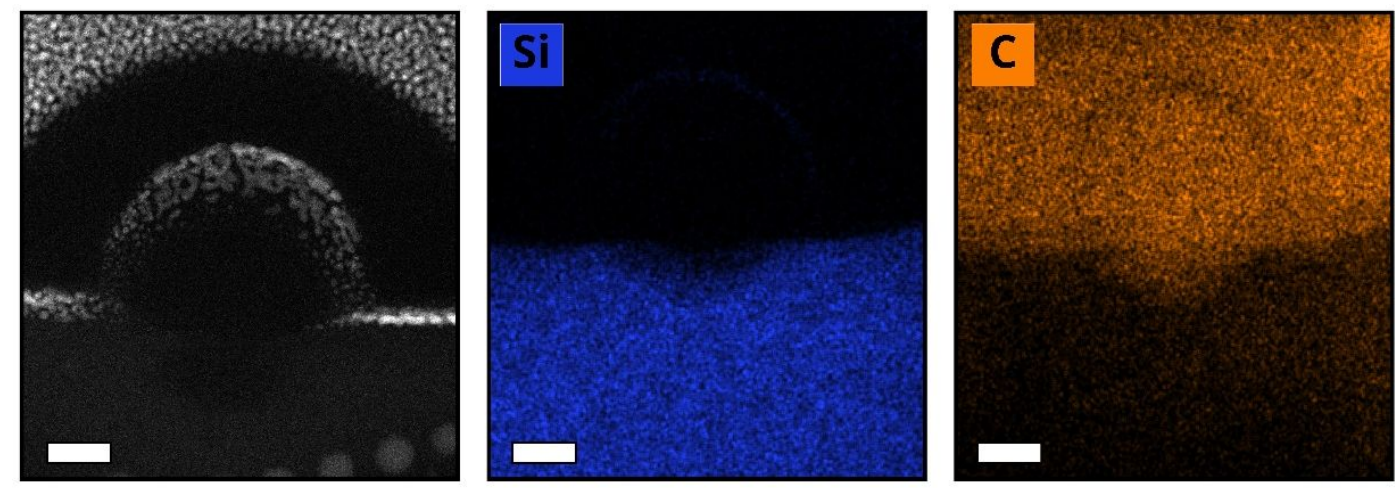

Figure S3. STEM-HAADF image of a lamellar cross-section containing latex NPs after spin-coating of sol-gel, and the corresponding Si/C elemental analysis. Scale bar: $20 \mathrm{~nm}$. 

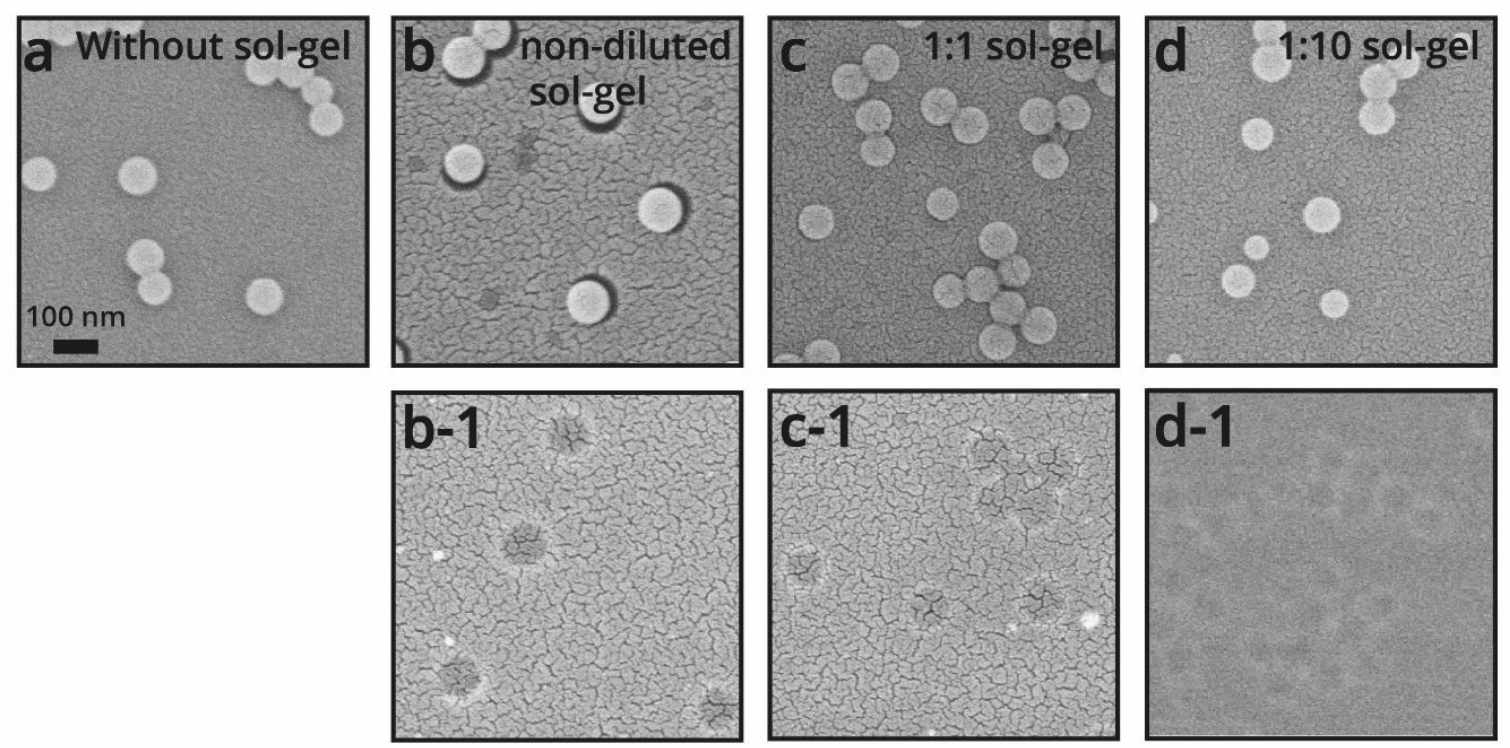

Figure S4. SEM images of a glass surface coated with latex NPs and sol-gel with different concentrations: (a) Without sol-gel; (b) non-diluted sol-gel; (c) 1:1 (d) 1:10 sol-gel solution diluted with ethanol:water 7:1 v/v mixture. (b-1), (c-1) and (d-1) After thermal treatment of the respective surfaces to remove the latex NPs. All samples were sputtered for $60 \mathrm{sec}$ with a thin AuPd layer.
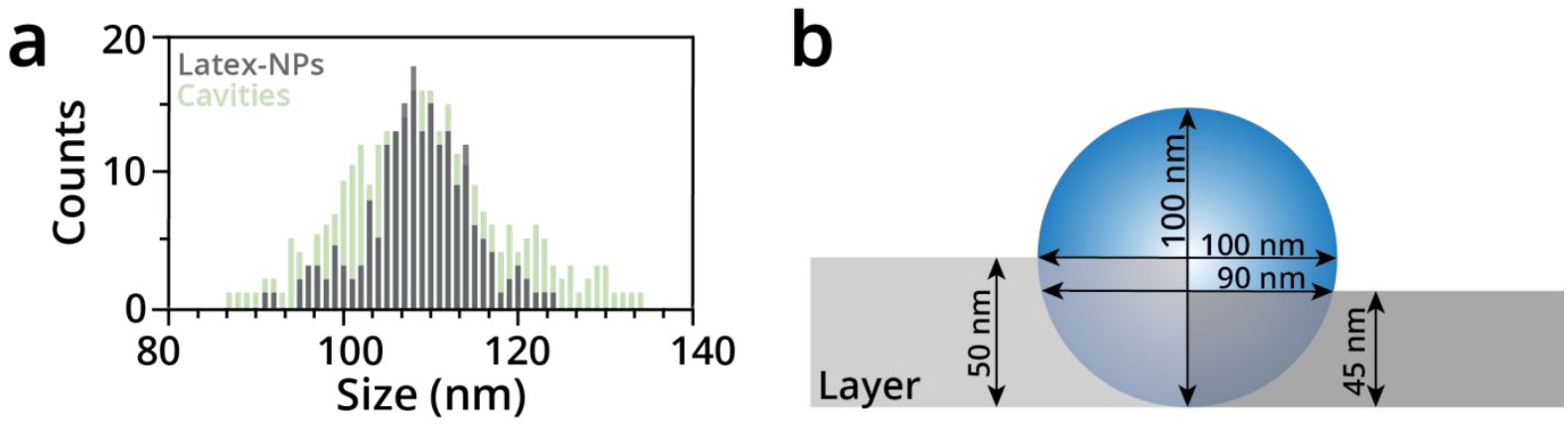

Figure S5. (a) Histograms of latex-NPs (grey) and cavities (green) size distribution determined by SEM images (the size of the cavities was multiplied by 1.1, see b). (b) Schematic illustration of the calculation of the nanoparticle diameter fitness based on the layer thickness. A $100 \mathrm{~nm}$ NP embedded in a $45 \mathrm{~nm}$ thickness layer will result in a cavity of $90 \mathrm{~nm}$ in diameter. Therefore, the size of the NP that fits into a $90 \mathrm{~nm}$ diameter cavity should be multiplied by 1.1 . 


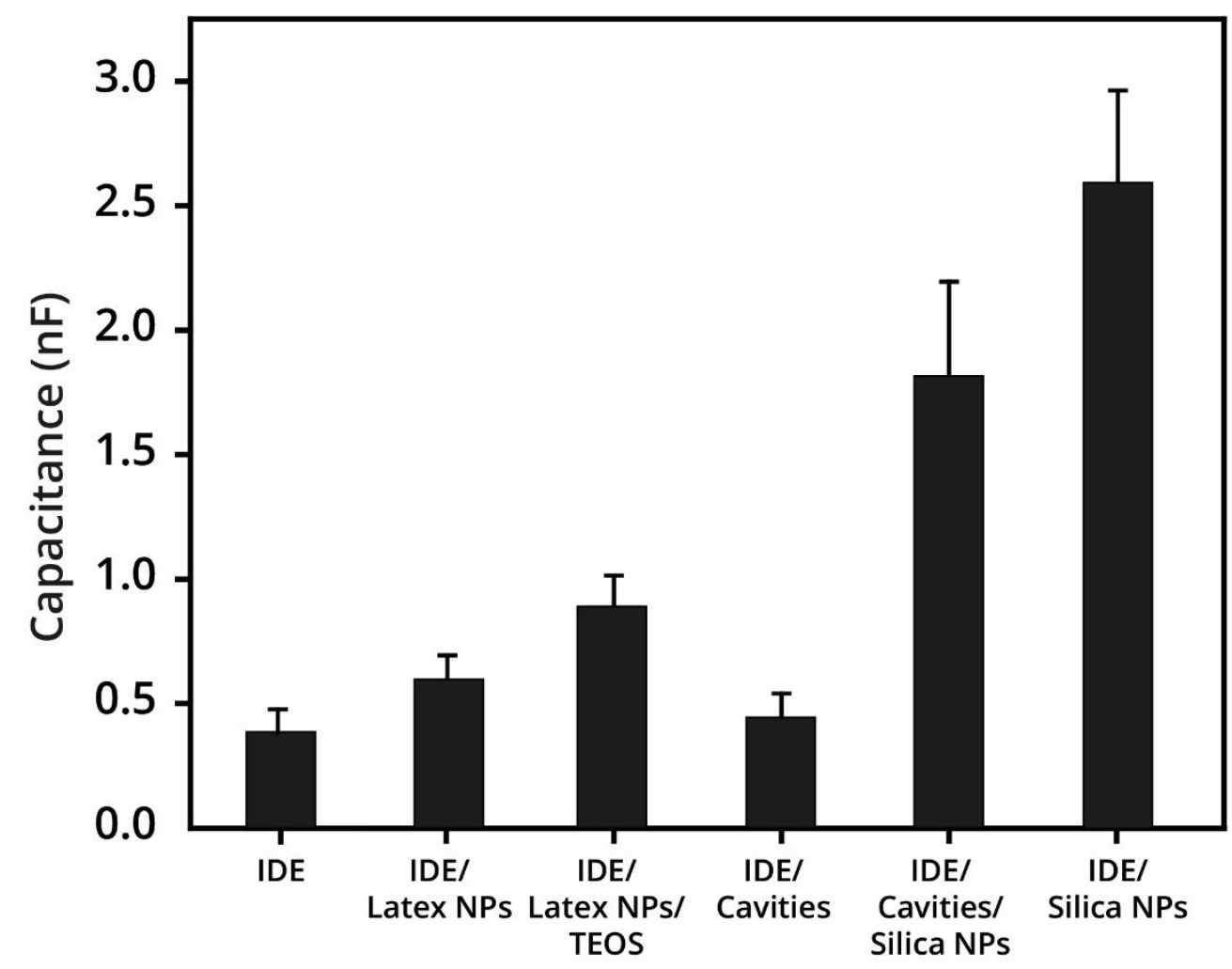

Figure S6. The capacitance value of the different stages for the NAIM-IDE formation and sensing.

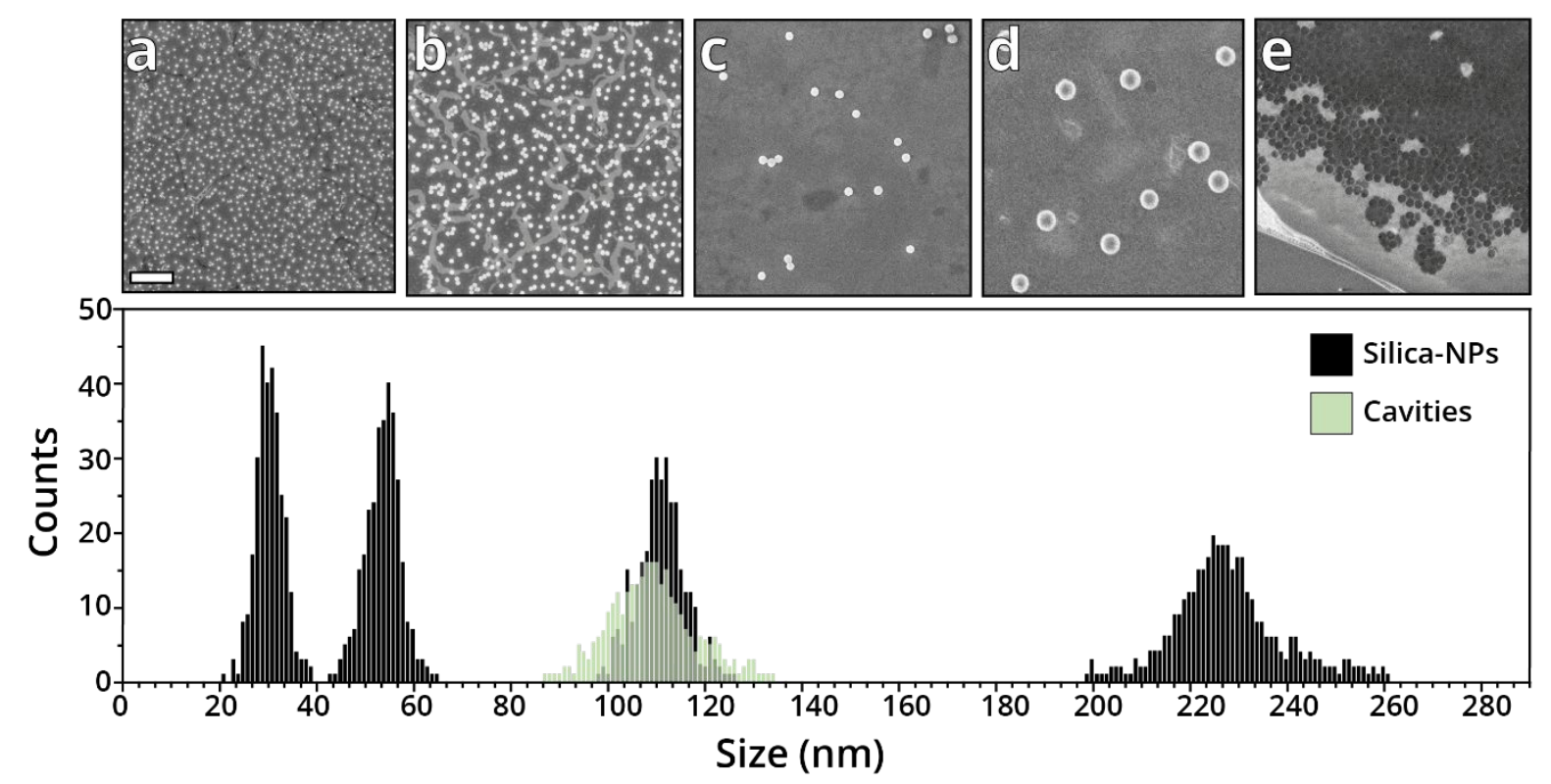

Figure S7. SEM images and histograms of particle and cavities size distribution determined by sizing the NPs/cavities from their SEM images. (a) 30, (b) 50, (c) 100 and (d) $200 \mathrm{~nm}$-sized Silica-NPs. (e) SEM image of $100 \mathrm{~nm}$ cavities. Each histogram was calculated from at least 300 particles. Scale bar: $500 \mathrm{~nm}$. 


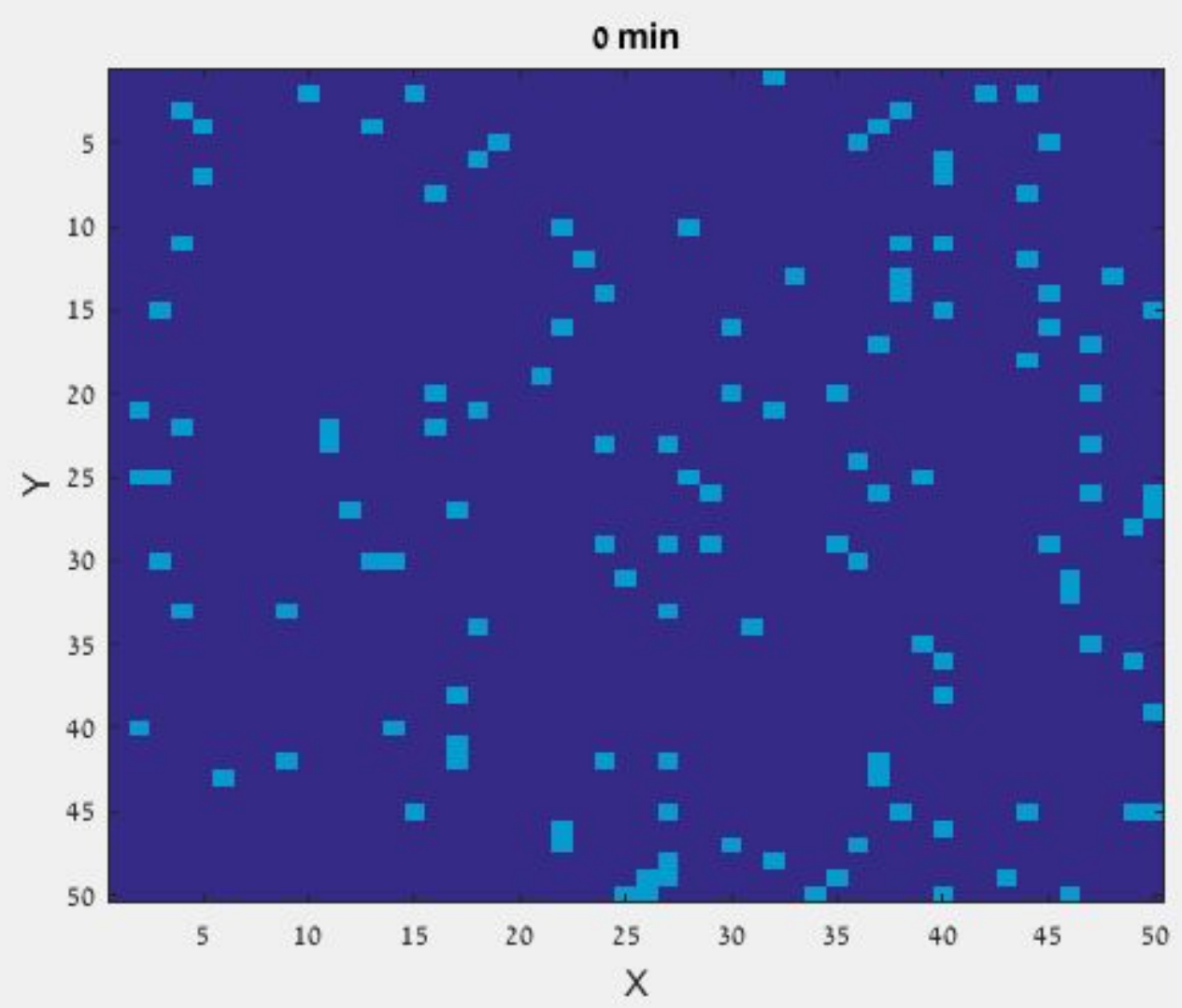

\section{https://i.imgur.com/W7gn167.gif}

Figure S8. A GIF animation showing the different time shots of the simulation.

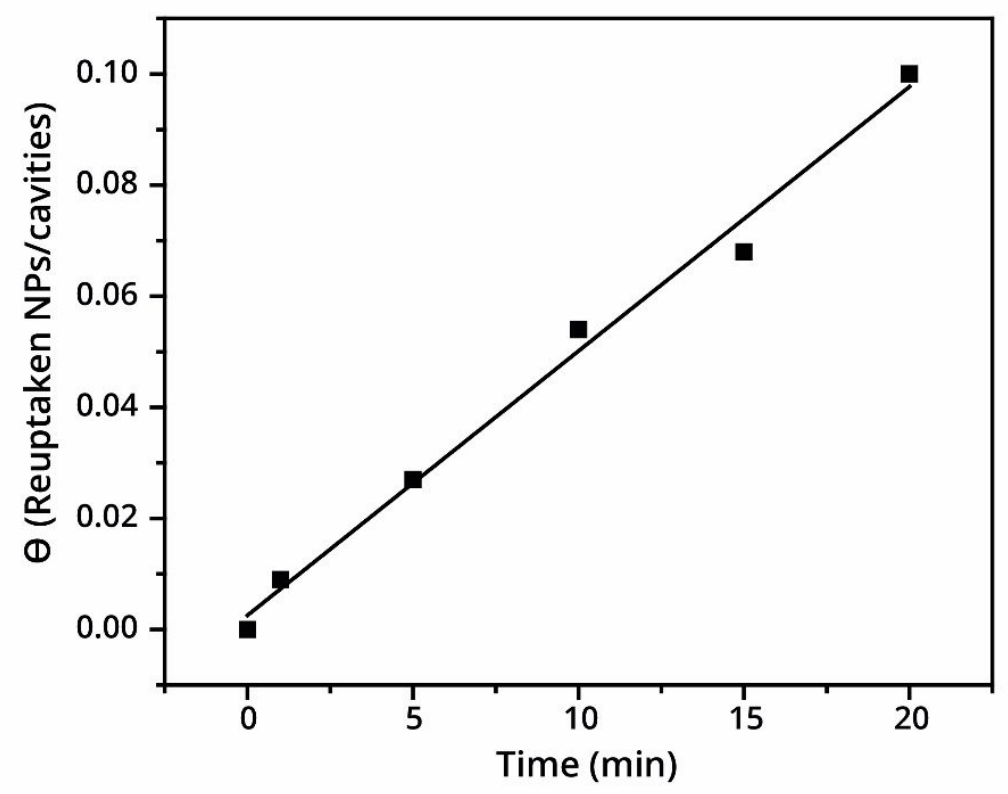

Figure S9. $\mathrm{SiO}_{2} \mathrm{NPs}$ reuptaken, divided by the average number of cavities that were observed by SEM images, as a function of exposure time to silica NPs aerosol. Linear fitting with R-Square equals 0.988 . 
